To appear in Molecular Physics

Vol. 00, No. 00, Month 200x, 1-20

\title{
ARTICLE
}

\section{A Global Potential Energy Surface for $\mathbf{H}_{3}^{+}$}

\author{
Irina I. Mizus ${ }^{1}$, Oleg L. Polyansky, ${ }^{1,2, \dagger}$ Laura K. McKemmish ${ }^{2,3}$ Jonathan Tennyson, ${ }^{2, *}$, \\ Alexander Alijah ${ }^{4}$ and Nikolai F. Zobov ${ }^{1}$, \\ ${ }^{1}$ Institute of Applied Physics, Russian Academy of Sciences, Ulyanov Street 46, Nizhny \\ Novgorod, Russia 603950 \\ ${ }^{2}$ Department of Physics and Astronomy, University College London, Gower Street, \\ London WC1E 6BT, UK \\ ${ }^{3}$ Department of Chemistry, University of New South Wales, Australia \\ ${ }^{4}$ Groupe de Spectrométrie Moléculaire et Atmosphérique, GSMA, UMR CNRS 7331, \\ Université de Reims Champagne-Ardenne, France \\ (Received 00 Month 200x; final version received 00 Month 200x)
}

\begin{abstract}
A globally correct potential energy surface (PES) for the $\mathrm{H}_{3}^{+}$molecular ion is presented. The Born-Oppenheimer (BO) ab initio grid points of Pavanello et al. [J. Chem. Phys. 136, 184303 (2012)] are refitted as BOPES75K, which reproduces the energies below dissociation with a root mean square deviation of $0.05 \mathrm{~cm}^{-1}$; points between dissociation and $75000 \mathrm{~cm}^{-1}$ are reproduced with the average accuracy of a few wavenumbers. The new PES75K+ potential combines BOPES75K with adiabatic, relativistic and quantum electrodynamics (QED) surfaces to provide the most accurate representation of the $\mathrm{H}_{3}^{+}$global potential to date, overcoming the limitations on previous high accuracy $\mathrm{H}_{3}^{+}$PESs near and above dissociation. $\mathrm{PES75K}+$ can be used to provide predictions of bound rovibrational energy levels with an accuracy of approaching $0.1 \mathrm{~cm}^{-1}$. Calculation of rovibrational energy levels within PES75K+ suggests that the non-adiabatic correction remains a limiting factor. The PES is also constructed to give the correct asymptotic limit making it suitable for use in studies of the $\mathrm{H}^{+}+\mathrm{H}_{2}$ prototypical chemical reaction. An improved dissociation energy for $\mathrm{H}_{3}^{+}$is derived as $D_{0}=35076 \pm 2 \mathrm{~cm}^{-1}$.
\end{abstract}

Keywords: ab initio, potential energy surface, dissociation, spectroscopy

† o.polyansky@ucl.ac.uk

*j.tennyson@ucl.ac.uk

ISSN: 0040-5167 print/ISSN 1754-2278 online

(c) 200x Taylor \& Francis

DOI: 10.1080/0040516YYxxxxxxxx

http://www.informaworld.com 


\section{Introduction}

The $\mathrm{H}_{3}^{+}$ion provides a benchmark system for two areas of science, which, up to now, have remained unrelated: the high accuracy, ab initio prediction of rotationvibration spectra 1] and reaction dynamics [2]. In fact, the two regimes are linked through the near-dissociation spectrum of Carrington and co-workers [3 8], which provides a direct connection between spectroscopy and dissociation dynamics. Theoretical studies to elucidate this spectrum [9], as well as studies, which try to model ultra-low energy $\mathrm{H}^{+}+\mathrm{H}_{2}$ reactive and non-reactive scattering [10 12], require surfaces of spectroscopic accuracy to recover the full resonance structure.

A number of global potential energy surfaces (PESs) are available for $\mathrm{H}_{3}^{+}$in its electronic ground state [13-23]. Of these we particularly note the GLH3P PES of Pavanello et al. [21], which is based on ab initio calculations of spectroscopic accuracy [24, 25], and the surface of Velilla et al. 18], which is based on less accurate $a b$ initio calculations but whose full treatment of the long-range proton $-\mathrm{H}_{2}$ interactions is vital for the study of ultra-low energy collisions [12].

The ab initio calculations of Pavanello et al. [21] used optimized explicitly correlated shifted Gaussian functions and were performed for an extensive grid of 41655 $\mathrm{H}_{3}^{+}$geometries. The calculations have an absolute accuracy of about $0.01 \mathrm{~cm}^{-1}$ for the non-relativistic, fixed-nuclei, electronic energy [26]; Pavanello et al.'s analytical fit reproduces these points below dissociation with a root mean square error of about $0.05 \mathrm{~cm}^{-1}$. However, certain asymptotic configurations were omitted from this fit as their inclusion led to significant deterioration in the accuracy at low energies. Therefore, these points are not well represented by the analytical GLH3P yielding unphysical features as shown in the supplemental material of the original paper [21]. Such unphysical features also appear in other global $\mathrm{H}_{3}^{+}$PESs [15]. Importantly, we note that the PES of Velilla et al. [18] does not show any unphysical behaviour.

The work of Pavanello et al. [21] also included an adiabatic or diagonal BornOppenheimer correction, but did not provide a global fitted surface for this effect. However, to make a PES spectroscopically accurate, global adiabatic, relativistic and QED (quantum electrodynamics) correction surfaces should also be included. Such surfaces are available [1]. In the present work, we report a significantly improved fit to the ab initio points of Pavanello et al. [21] in the high-energy region, so that a very accurate global PES is obtained, which includes BO energies, QED, 
relativistic and adiabatic corrections. This surface, which we call PES75K+, is suitable for both the computation of predissociation energies and line positions, as well as for accurate reactive and non-reactive scattering calculations.

The present paper is organized as follows: In section 2 we describe the functional form of the improved global $\mathrm{H}_{3}^{+}$PES. The global PES obtained as an analytical form fitted to the accurate $a b$ initio points is analyzed in section 3 , while section 4 contains a comparison of the rovibrational energy level calculations using the present surface and our previous one. Section 5 concludes our paper.

\section{Structure of the new global potential}

\subsection{Born-Oppenheimer part of the potential}

Initially we attempted to fit the $a b$ initio data of Pavanello et al. [21] using a single functional form. However, our attempts failed to give a satisfactory fit. We there adopted an alternative approach where the BO part of the new ab initio highaccuracy global potential energy surface for the $\mathrm{H}_{3}^{+}$molecule consists of three independent parts: low and high energy short-range potentials joined together smoothly at $42000 \mathrm{~cm}^{-1}$ using the energy switching approach of Varandas [27], and an analytic long-range part, which gives the correct asymptotic behaviour. The lower part of the short-range potential, $V_{\text {low }}$, consists of the three-body terms from the GLH3P potential obtained by Pavanello et al. [21] with a correction in the energy region where the GLH3P surface is not accurate. The upper part, $V_{\text {up }}$, is an analogous adaptation of the PES of Velilla et al. [18]. The long-range potential is based on the analytic form used by Velilla et al. [18]. We call the resulting PES BOPES75K. Figure 1 illustrates the structure of BOPES75K.

The BOPES75K potential extensively utilizes switching functions,

$$
f\left(t-t_{0}, \alpha\right)=\frac{1}{1+e^{-2 \alpha \Delta t}}=\frac{1}{2}[1+\tanh (\alpha \Delta t)]
$$

where $\alpha$ is a parameter that controls the sharpness of the switch (large $\alpha$ is a fast switch and small $\alpha$ is a slow switch), $t_{0}$ is the switching point in variable $t$ and $\Delta t=t-t_{0}$ is the distance from the position of the switch. However, it is often easier to set a width for the switching zone, $b$, so that at the edges of the region

$\left(t_{0}-\frac{b}{2}, t_{0}+\frac{b}{2}\right)$ the switching function would reach its asymptotic values (0 or 1 , 


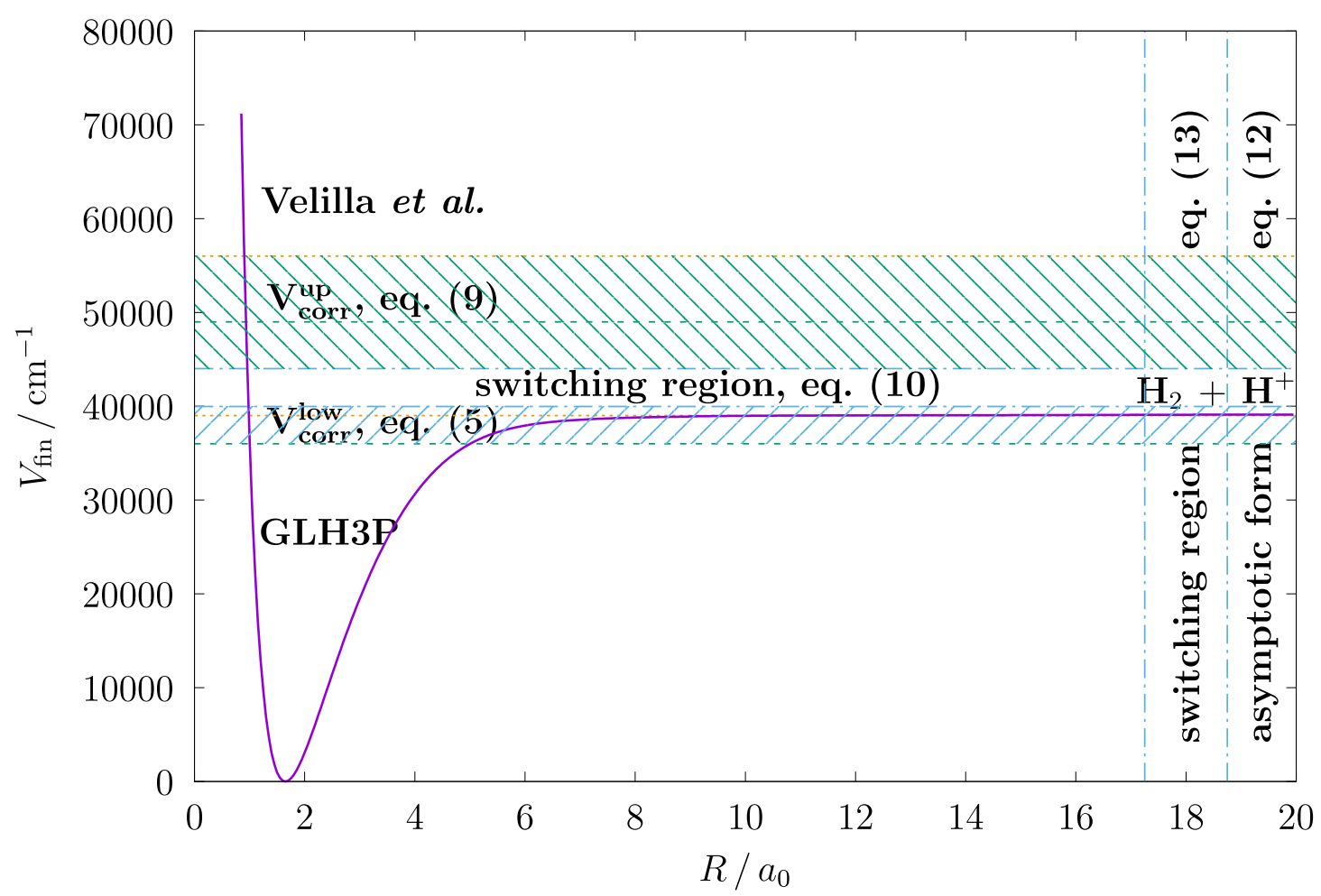

Figure 1. Illustration of the structure of new BOPES75K potential energy surface. The curve corresponds to isosceles configurations of $\mathrm{H}_{3}^{+}$with the third bond length fixed at its equilibrium value $1.65 a_{0}$. Dashed lines mark the borders of regions, where the GLH3P PES (the green line) and the potential of Velilla et al. (the yellow line) were corrected, and the blue dashed lines denote the borders of switching regions. The shaded domains correspond to the parts of corrected potentials, which don't fall into the energy switching region.

correspondingly) with an accuracy of $\delta$ :

$$
\begin{gathered}
f\left(t_{0}+\frac{b}{2}\right)=1-\frac{\delta}{100}, \\
f\left(t_{0}-\frac{b}{2}\right)=\frac{\delta}{100},
\end{gathered}
$$

where $\delta$ is expressed as a percentage. So, the sharpness of the switch is fully defined by the width of the switching zone and the accuracy $\delta$ and can be expressed as

$$
\alpha=\ln \left(\frac{100}{\delta}-1\right) / b
$$

We will use the parameters $t_{0}, b$ and $\delta$ later as variables in some optimization procedures.

A product of two switching functions can be used to smoothly turn on and off a term within a finite region by using oppositely signed switching parameters. A 
similar approach with multiple switchings was applied to the $\mathrm{NO}_{2}$ molecule by Varandas in [28]. In this work we use switching functions based directly on the coordinates and on the value of the potential energy at a given geometry. In the latter case it is necessary to map from the given coordinates to a value of the PES at that point in order to evaluate the switching function. Below $V_{d}$ denotes the value of this "distributing" potential; where necessary the global $\mathrm{H}_{3}^{+}$potential of Velilla et al. [18] was used to evaluate $V_{d}$.

The correction to $V_{\text {low }}$ was made by first approximating the differences between the GLH3P energy values and the corresponding ab initio energy values at 2414 $a b$ initio geometries in the range 35000 to $42000 \mathrm{~cm}^{-1}$ using a polynomial

$$
V_{\text {poly }}^{X}=\sum_{n m k}^{N} V_{n m k}^{X} S_{a}^{n} S_{e}^{2 m+3 k} \cos (3 k \varphi), n+2 m+3 k \leq N,
$$

where the superscript $X$ on $V_{\text {poly }}^{X}$ and $V_{n m k}^{X}$ is used to distinguish the various expansions, in this case $X=$ low for the low-energy short-range PES and $N=$ 31 coefficients with a maximum degree of 7 . This expression uses the following symmetrized coordinates:

$$
\begin{array}{ll}
S_{a}=\left(\Delta R_{12}+\Delta R_{23}+\Delta R_{31}\right) / \sqrt{3}, & \\
S_{x}=\left(2 \Delta R_{12}-\Delta R_{23}-\Delta R_{31}\right) / \sqrt{6} & =S_{e} \cos \varphi, \\
S_{y}=\left(\Delta R_{23}-\Delta R_{31}\right) / \sqrt{2} & =S_{e} \sin \varphi,
\end{array}
$$

where $\Delta R_{i k}=R_{i k}-R_{e}$ is the displacement from the equilibrium value of $R_{e}=$ $1.65 a_{0}$ in the bond length between the $i$-th and $k$-th protons in $\mathrm{H}_{3}^{+}$and the angle $\varphi$ from eq. (3) can be obtained from the latter equations as $\varphi=\arctan \left(\frac{S_{y}}{S_{x}}\right)$. This analytical form reflects correctly the $D_{3 h}$ symmetry of the $\mathrm{H}_{3}^{+}$molecular system. The standard deviation of the approximated energy differences from the numerical values of the polynomial $V_{\text {poly }}^{\text {low }}$ in the corresponding ab initio geometries is about $2.3 \mathrm{~cm}^{-1}$.

We added the possibility of smoothly switching on and off this polynomial term by multiplying it with switching factors $f_{\text {low }}$ and $f_{\text {up }}$ :

$$
V_{\text {corr }}^{\text {low }}=V_{\text {poly }}^{\text {low }} f_{\text {low }} f_{\text {up }}
$$


where

$$
\begin{aligned}
f_{\text {low }} & =\frac{1}{1+e^{-2 \alpha_{l}\left(V_{d}-E_{l}\right)}}, \\
f_{\text {up }} & =\frac{1}{1+e^{2 \alpha_{u}\left(V_{d}-E_{u}\right)}}, \\
\alpha_{l, u} & =\ln \left(\frac{100}{\delta}-1\right) / b_{l, u} .
\end{aligned}
$$

Here and elsewhere $\delta=0.1 \%$, and $b_{u}, b_{l}, E_{u}$ and $E_{l}$ are the adjustable nonlinear parameters of the fit. The lower part of the potential is thus represented as

$$
V_{\mathrm{low}}=V_{\mathrm{GLH} 3 \mathrm{P}}-V_{\mathrm{corr}}^{\mathrm{low}}
$$

The upper part of our new global $\mathrm{H}_{3}^{+} \mathrm{BO}$ PES is based on the potential of Velilla et al. [18], denoted $V_{\text {Vel. }}$, as the GLH3P potential is not reliable in this energy region. It is corrected in the same way as the lower part:

$$
V_{\text {up }}=V_{\text {vel. }}-V_{\text {corr }}^{\text {up }}
$$

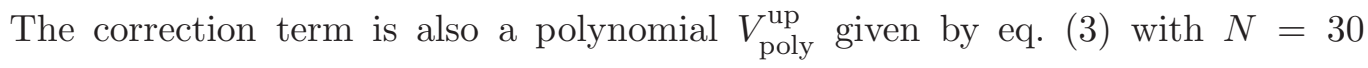
coefficients $V_{n m k}^{\text {up }}$ and a maximum degree of 7 . This function approximates a set of 4582 differences between the $V_{\text {Vel. }}$ values and the accurate $a b$ initio energies in the region from $40000 \mathrm{~cm}^{-1}$ to $55000 \mathrm{~cm}^{-1}$ with a standard deviation of about $4.6 \mathrm{~cm}^{-1}$. Again the function switches on and off

$$
V_{\text {corr }}^{\text {up }}=V_{\text {poly }}^{\text {up }} f_{\text {low }} f_{\text {up }}
$$

where $f_{\text {low }}$ and $f_{\text {up }}$ are also given by eq. (6), just like the ones used to describe $V_{\text {corr }}^{\text {low }}$, but with their own values of adjustable nonlinear parameters.

The two parts were then merged using the energy switching scheme [27] to yield a composite potential

$$
V_{\text {comp }}=\varphi_{\text {low }} V_{\text {low }}+\varphi_{\text {up }} V_{\text {up }}
$$

where

$$
\begin{aligned}
\varphi_{\text {low }} & =\frac{1}{2}\left(1+\tanh \left(-\gamma\left(V_{u p}-E_{0}\right)\right),\right. \\
\varphi_{\text {up }} & =\frac{1}{2}\left(1+\tanh \left(\gamma\left(V_{u p}-E_{0}\right)\right),\right. \\
\gamma & =\tilde{g}_{0}+\tilde{g}_{1}\left(V_{u p}-E_{0}\right)^{2},
\end{aligned}
$$


and the coefficients $\tilde{g}_{0,1}=g_{0,1} /\left(100 k^{2}\right)$, where $k=219474.631 \mathrm{~cm}^{-1} E_{h}^{-1}$ is a wellknown conversion factor of energy from $E_{h}$ to $\mathrm{cm}^{-1}$ units.

The leading term in the long-range behaviour of the $\mathrm{H}_{3}^{+}$potential at its first dissociation limit into $\mathrm{H}_{2}+\mathrm{H}^{+}$is correctly represented in the GLH3P potential, as it is derived from the analytical potentials of Viegas et al. [17], and therefore also in $V_{\text {comp }}$. However, the full, angularly-dependent asymptotic behaviour is given by the multipole expansion [18, 29]

$V-D_{e} \underset{R \rightarrow \infty}{\approx} \frac{Q_{2}(r) P_{2}(\cos \theta)}{R^{3}}-\frac{\frac{1}{2} a_{0}(r)+\frac{1}{3}\left[\alpha_{\|}(r)-\alpha_{\perp}(r)\right] P_{2}(\cos \theta)}{R^{4}}+\cdots+V_{H_{2}}(r)$,

where $r$ is the internuclear separation of the diatomic (taken as the shortest distance between two of the nuclei), $R$ is the distance between the midpoint between these two nuclei and the third nucleus, and $\theta$ is the angle between $r$ and $R$. In the case where two nearest nuclei are the same, these coordinates are standard Jacobi coordinates. We enforce this behaviour in the asymptotic region by explicitly joining our BO surface defined in eq. (10) to the one due to Velilla et al., where this form is implemented. This is done at a certain distance $R_{0}$ using the switching procedure

$$
V_{\text {fin }}=f_{\text {short }} V_{\text {comp. }}+f_{\text {long }} V_{\text {Vel. }}
$$

where

$$
\begin{gathered}
f_{\text {long }}=\frac{1}{1+e^{-2 \alpha\left(R-R_{0}\right)}}, \\
f_{\text {short }}=\frac{1}{1+e^{2 \alpha\left(R-R_{0}\right)}}
\end{gathered}
$$

and $\alpha$ is given by eq. (2) $\mathrm{R}_{0}$ and $b$ are two further nonlinear parameters in our final BO PES $V_{\text {fin }}$, and $\delta$ has the same value as earlier, see eq. (6). This switching also ensures that the $\mathrm{H}_{2}$ diatomic potential is correctly reproduced by the $V_{\text {fin }}$ potential as $R \rightarrow \infty$.

Thus, the final form of our global BO PES contains 61 linear and 15 nonlinear adjustable parameters of the fit, including the parameters $E_{0}, g_{0}$ and $g_{1}$ for the energy switching [27]. The linear parameters were determined by least-squares fitting; the nonlinear ones were adjusted manually by a trial-and-error procedure. Their final values are summarized in Table 1 and Table 2 . 
Table 1. Adjustable nonlinear parameters used to define our new global ab initio PES for the $\mathrm{H}_{3}^{+}$molecule together with the number $N_{\text {coef }}$ of linear polynomial parameters; all polynomials have a maximum degree of 7 .

\begin{tabular}{|c|c|c|c|c|c|c|c|c|c|c|c|}
\hline Part of the PES & $N_{\text {coef }}$ & $\delta$ & $b_{u} / \mathrm{cm}^{-1}$ & $b_{l} / \mathrm{cm}^{-1}$ & $E_{u} / \mathrm{cm}^{-1}$ & $E_{l} / \mathrm{cm}^{-1}$ & $E_{0} / \mathrm{cm}^{-1}$ & $g_{0}$ & $g_{1}$ & $\mathrm{R}_{0} / a_{0}$ & $b / a_{0}$ \\
\hline Lower (GLH3P-like) & 31 & 0.1 & 300 & 100 & 48000 & 37000 & \multirow{2}{*}{42000} & \multirow{2}{*}{1.71} & \multirow{2}{*}{2000} & \multirow{2}{*}{18} & \multirow{2}{*}{1} \\
\hline Upper (Velilla et al.-like) & 30 & 0.1 & 1400 & 80 & 55000 & 40000 & & & & & \\
\hline
\end{tabular}

\section{2. $\quad$ Correction surfaces}

It is necessary to also take into account adiabatic, relativistic and QED corrections to the BO approximation. The final form of our potential PES75K+ was obtained by addition of these correction surfaces to the BOPES75K PES.

All correction surfaces consist of two parts: a polynomial given in the form of eq. (3), and an exponential damping function, which prevents unphysical behaviour of the corrections for geometries with large internuclear distances.

\subsubsection{Relativistic and QED correction surfaces}

The polynomial parts $V_{\text {poly }}^{\text {rel, QED }}$ of relativistic and QED correction surfaces have the analytical form of eq. (3), but different sets of adjustable parameters: $N=52$ for the relativistic correction, which correspond to maximum polynomial power of 10 , and $N=44$ coefficients $V_{n m k}^{\mathrm{QED}}$ for the QED correction, with maximum polynomial power of 9 . These sets of parameters were obtained from two fits at points with energies below $38000 \mathrm{~cm}^{-1}$ : (i) a fit of 3380 relativistic points by Bachorz et al. 30] to $V_{\text {poly }}^{\text {rel }}$, which are reproduced with a standard deviation (rms) value of $0.008 \mathrm{~cm}^{-1}$, and (ii) a fit of $6413 \mathrm{QED}$ points by Lodi et al. 31] to $V_{\text {poly }}^{\text {QED }}$ with the rms deviation of $0.001 \mathrm{~cm}^{-1}$.

Our estimates show that the overall effect of relativistic and QED corrections on $\mathrm{H}_{3}^{+}$energy states even in the near-dissociation region is only about $0.1 \mathrm{~cm}^{-1}$, which is much smaller than the corresponding BOPES75K accuracy of a few $\mathrm{cm}^{-1}$, and we can neglect this effect for states with large internuclear distances in the region of the first dissociation limit and above.

We included the relativistic and the QED correction surfaces using a combined polynomial form $V_{\text {poly }}^{\text {rel }}{ }^{\text {QED }}$ given by eq. (3), with maximum power of 10 and polynomial coefficients $V_{n m k}^{\mathrm{rel}+\mathrm{QED}}=V_{n m k}^{\mathrm{rel}}+V_{n m k}^{\mathrm{QED}}$. The combined polynomial correction $V_{\text {poly }}^{\mathrm{rel}+\mathrm{QED}}=V_{\text {poly }}^{\mathrm{rel}}+V_{\text {poly }}^{\mathrm{QED}}$ is then complemented by an exponentially decreasing term, which smoothly switches the combined correction off when its value becomes too large because of increasing internuclear distances: 
Table 2. Coefficients of the polynomials $V_{\text {poly }}^{\text {low }}$ and $V_{\text {poly }}^{\text {up }}$ given by eq. (3) for the correction terms to the lower and the upper parts of the new BOPES75K potential, correspondingly.

\begin{tabular}{rrrrr}
\hline \hline$n$ & $2 m$ & $3 k$ & $V_{n m k}^{\text {low }}$ & $V_{n m k}^{\text {up }}$ \\
\hline 0 & 0 & 0 & -0.75143071 & 28.89617715 \\
1 & 0 & 0 & -1.76440106 & -10.58157805 \\
2 & 0 & 0 & -0.09859390 & 2.87604608 \\
0 & 2 & 0 & 0.99200748 & 2.08628333 \\
3 & 0 & 0 & 1.48567895 & -0.62636049 \\
1 & 2 & 0 & -4.46772673 & -0.51256565 \\
0 & 0 & 3 & -3.90622308 & -1.15962111 \\
4 & 0 & 0 & -0.37926323 & 0.13476748 \\
2 & 2 & 0 & 1.53719611 & -0.08342574 \\
1 & 0 & 3 & 2.48855132 & 0.29409500 \\
0 & 4 & 0 & 1.37657184 & 0.10614684 \\
5 & 0 & 0 & -0.01544350 & -0.02831679 \\
3 & 2 & 0 & 0.07169542 & 0.08313826 \\
2 & 0 & 3 & -0.37054284 & -0.01860622 \\
1 & 4 & 0 & -0.68993265 & -0.10208867 \\
0 & 2 & 3 & -0.20471568 & -0.03815698 \\
6 & 0 & 0 & 0.00037056 & 0.00295364 \\
4 & 2 & 0 & 0.00167004 & -0.01213435 \\
3 & 0 & 3 & 0.00764265 & 0.00056655 \\
2 & 4 & 0 & -0.00024218 & 0.01681600 \\
1 & 2 & 3 & 0.02173168 & 0.00053141 \\
0 & 6 & 0 & 0.04686634 & -0.00595500 \\
0 & 0 & 6 & -0.00943583 & -0.00116108 \\
7 & 0 & 0 & 0.00077371 & -0.00010578 \\
5 & 2 & 0 & -0.00681003 & 0.00050616 \\
4 & 0 & 3 & -0.00285356 & -0.00000479 \\
3 & 4 & 0 & 0.01863322 & -0.00079326 \\
2 & 2 & 3 & 0.01297023 & 0.00002889 \\
1 & 6 & 0 & -0.01472313 & 0.00040228 \\
1 & 0 & 6 & 0.00114263 & 0.00004892 \\
0 & 4 & 3 & -0.01094351 & \\
\hline \hline & & & &
\end{tabular}

$$
\begin{aligned}
V_{\mathrm{rel}+\mathrm{QED}} & =V_{\mathrm{poly}}^{\mathrm{rel}+\mathrm{QED}} \cdot h \\
h & =\frac{1}{1+e^{2 \alpha_{c}\left(\left|V_{\mathrm{poly}}^{\mathrm{rel}+\mathrm{QED}}\right|-V_{0}\right)}} \\
\alpha_{c} & =\ln \left(\frac{100}{\delta}-1\right) / b_{c}
\end{aligned}
$$

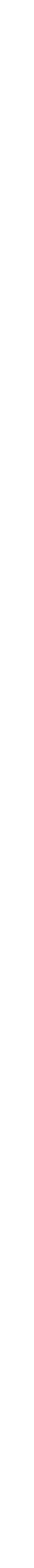

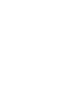

s.


where $\delta=0.1, b_{c}=3 \mathrm{~cm}^{-1}, V_{0}=6 \mathrm{~cm}^{-1}$.

\subsubsection{Adiabatic correction surface}

The present adiabatic correction surface is a slightly modified version of the one computed by us previously [1]. Its polynomial part $V_{\text {poly }}^{\text {ad }}$ has again the analytical form of eq. (3), with a set of 78 non-zero coefficients $V_{n m k}^{\mathrm{ad}}$ and a maximum polynomial power of 12 , but in this case transformed coordinates

$$
\tilde{R}_{i j}=\left[1-e^{-\beta\left(R_{i j} / R_{e}-1\right)}\right] / \beta
$$

with $\beta=1.3$ were used instead of the differences $\Delta R_{i j}$. Parameters $V_{\eta m p l}^{\text {ad }}$ were obtained by fitting 5591 adiabatic points computed by Pavanello et al. [21], corresponding to energies up to $38000 \mathrm{~cm}^{-1}$, to $V_{\text {poly }}^{\text {ad }}$. The resulting git gave an rms value of $0.116 \mathrm{~cm}^{-1}$.

Unlike relativistic and QED corrections, the adiabatic one has an effect on $\mathrm{H}_{3}^{+}$ energy states in the near-dissociation region, which is comparable with the corresponding BOPES75K accuracy. To extrapolate the adiabatic correction surface to the region above $38000 \mathrm{~cm}^{-1}$, we took an adiabatic point, which is close to the dissociation limit, and considered its value $V_{0}^{\text {ad }}=-114.5 \mathrm{~cm}^{-1}$ as a constant asymptote for $\mathrm{H}_{3}^{+}$molecular configurations with two internuclear distances greater than $R_{0}^{\mathrm{ad}}=10 a_{0}$ :

$$
\begin{aligned}
V_{\mathrm{ad}} & =V_{\mathrm{poly}}^{\mathrm{ad}} h_{\mathrm{low}}+V_{0}^{\mathrm{ad}} h_{\mathrm{up}}, \\
h_{\mathrm{low}} & =\frac{1}{1+e^{2 \alpha_{c}^{\mathrm{ad}}\left(R-R_{0}^{\mathrm{ad}}\right)}}, \\
h_{\mathrm{up}} & =\frac{1}{1+e^{-2 \alpha_{c}^{\mathrm{ad}}\left(R-R_{0}^{\mathrm{ad}}\right)}}, \\
\alpha_{c}^{\mathrm{ad}} & =\ln \left(\frac{100}{\delta}-1\right) / b_{c}^{\mathrm{ad}},
\end{aligned}
$$

where $\delta=0.1, b_{c}^{\text {ad }}=1 \mathrm{~cm}^{-1}$, and distance $R$ has the same meaning as earlier in eq. (12).

The Fortran files with BOPES75K and global correction surfaces together with files containing their polynomial constants are presented in the supplementary material. 


\section{Properties of the new global BO PES}

In this work we used a set of 40537 ab initio energies computed by Pavanello et al. [21], which span energies up to $75500 \mathrm{~cm}^{-1}$ and are reproduced by the new potential BOPES75K with a standard deviation value of $4.9 \mathrm{~cm}^{-1} .786$ points (about $2 \%$ from the total set of 41323 geometries) with energy values from $37090 \mathrm{~cm}^{-1}$ to $75380 \mathrm{~cm}^{-1}$ were excluded from our calculations, because their inclusion significantly deteriorates the accuracy. About $20 \%$ of them have two large (greater than $\left.7 a_{0}\right)$ internuclear distances and energies up to $42000 \mathrm{~cm}^{-1}$, and the others correspond to energies above $53000 \mathrm{~cm}^{-1}$ and have comparatively small bond lengths. The excluded points are nevertheless represented reasonably well by BOPES75K, with a standard deviation of $40.4 \mathrm{~cm}^{-1}$ and a maximum deviation of about $170 \mathrm{~cm}^{-1}$.

Table 3 and Figure 2 compare the GLH3P potential, the double many-body expansion (DMBE) potential of Viegas et al. [17], whose function form is the basis of the GLH3P PES, the PES by Velilla et al. and our new BOPES75K for different energy ranges. It is clear that our new BO PES retains the accuracy of the GLH3P fit at low energy while greatly improving its behaviour near and above dissociation.

Table 3. Standard deviations, in $\mathrm{cm}^{-1}$, of PES energy minus the ab initio value for the GLH3P potential 21], the DMBE potential of Viegas et al. 17], the PES by Velilla et al. 18] and the new BOPES75K potential for different energy ranges. The zero energy for all calculations was taken from the GLH3P potential. $N(E)$ denotes the number of ab initio points falling in the given energy range. The rms values indicated by a star* correspond to energy regions that are not covered by the GLH3P surface.

\begin{tabular}{crrrrr}
\hline \hline Energy range $/ \mathrm{cm}^{-1}$ & $N(E)$ & DMBE & GLH3P & Velilla et al. & BOPES75K \\
\hline $0-35000$ & 5422 & 30.56 & 0.0447 & 22.34 & 0.0447 \\
$0-37000$ & 6005 & 60.42 & 0.0512 & 22.37 & 0.0666 \\
$37000-42000$ & 1841 & 151.30 & 11.18 & 23.68 & 3.070 \\
$42000-45000$ & 934 & 121.90 & 27.65 & 21.38 & 5.611 \\
$45000-50000$ & 1503 & 120.71 & $34.99^{*}$ & 18.32 & 4.861 \\
$50000-55000$ & 1690 & 111.27 & $246.34^{*}$ & 17.10 & 4.981 \\
$53000-75500$ & 29260 & 84.12 & $1.4 \mathrm{E}+07^{*}$ & 5.872 & 5.443 \\
\hline \hline
\end{tabular}

Some two-dimensional cuts of our new global ab initio BOPES75K for $\mathrm{H}_{3}^{+}$are pictured in Fig. 3. They demonstrate the smooth behaviour of our new BO PES, in contrast to the analogous cuts through the GLH3P potential, which show serious unphysical features.

Some two-dimensional cuts and contour plots of our new global ab initio 


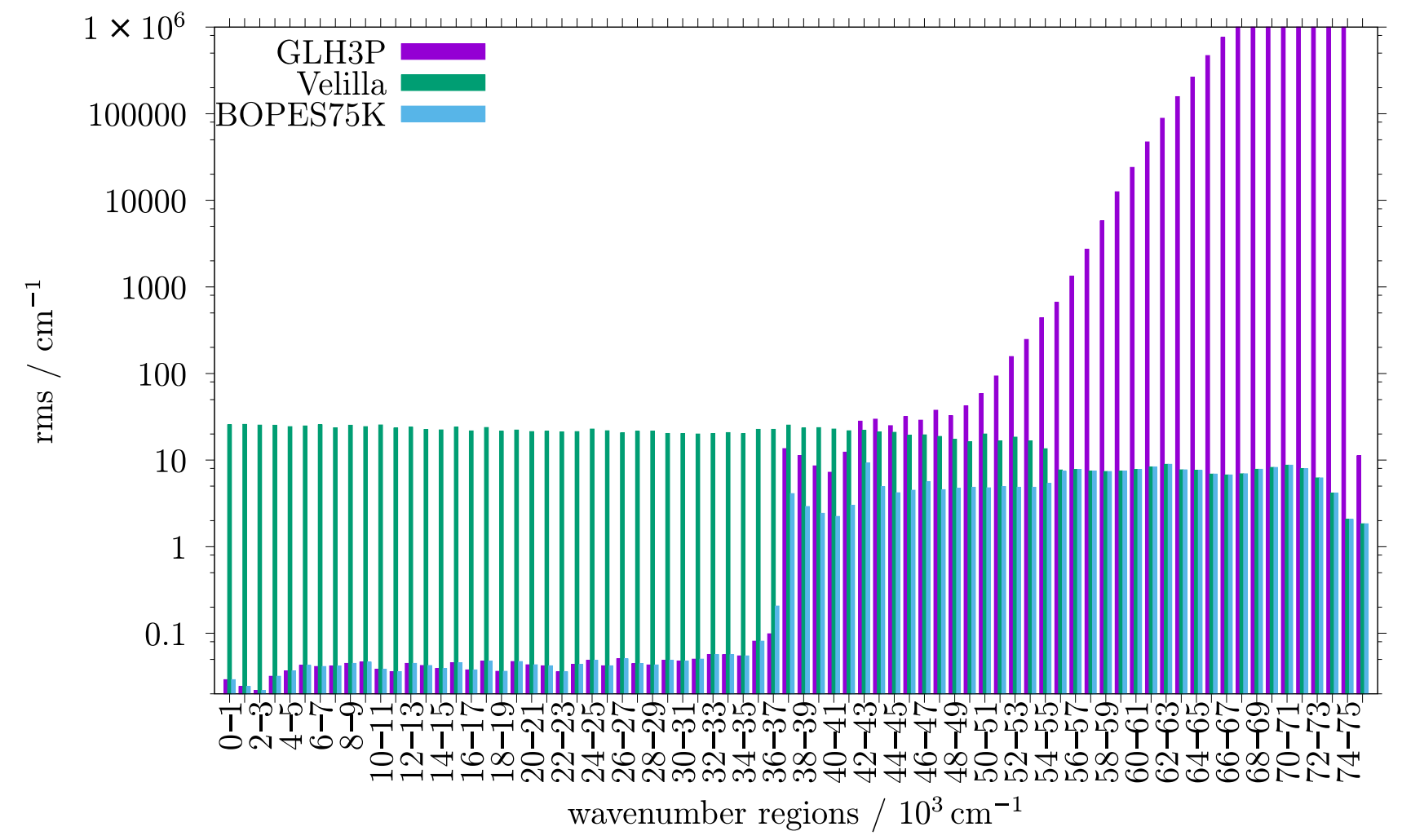

Figure 2. Standard deviations, in $\mathrm{cm}^{-1}$, with which various potentials reproduce the high accuracy $a b$ initio points computed by Pavanello et al. 21]: GLH3P potential 21], the PES by Velilla et al. [18] and the new BOPES75K potential. Energy ranges all have equal widths of $1000 \mathrm{~cm}^{-1}$.

BOPES75K for $\mathrm{H}_{3}^{+}$are shown in Fig. 3, which compares with the GLH3P potential, and Fig. 4 , which illustrates the behaviour in switching regions. The plots demonstrate the smooth behavior of our new BO PES; in contrast some of the analogous cuts through the GLH3P potential show serious unphysical features.

The new BOPES75K can be used to give the dissociation limit into $\mathrm{H}_{3}^{+} \rightarrow$ $\mathrm{H}_{2}+\mathrm{H}^{+} D_{e}=37195.3 \mathrm{~cm}^{-1}$. This is due to the procedure of obtaining the new BO potential presented in Sec. 2. For $\mathrm{H}_{2}-\mathrm{H}^{+}$distances larger than $R=19 a_{0}$, the absolute values of differences between the BOPES75K and the original potential by Velilla et al. are less than $0.005 \mathrm{~cm}^{-1}$. In particular, the PES of Velilla et al. reproduces a set of high-precision $a b$ initio points obtained by them in [18] with a standard deviation of only $1.80 \mathrm{~cm}^{-1}$ for $\mathrm{H}_{2}-\mathrm{H}^{+}$distances $R$ larger than $10 a_{0}$ in the region of the first dissociation limit, and gives a value of the $\mathrm{BO}$ dissociation energy $D_{e}=37170 \mathrm{~cm}^{-1}$.

The $\mathrm{BO}$ value of dissociation energy $D_{0}$ obtained on the basis of our new BOPES75K is $D_{0}=35011.8 \mathrm{~cm}^{-1}$. In this calculation an experimental value $E_{0}\left(\mathrm{H}_{2}\right)=2179.3 \pm 0.1 \mathrm{~cm}^{-1}$ for $\mathrm{H}_{2}$ vibrational zero-point energy (ZPE) was used 


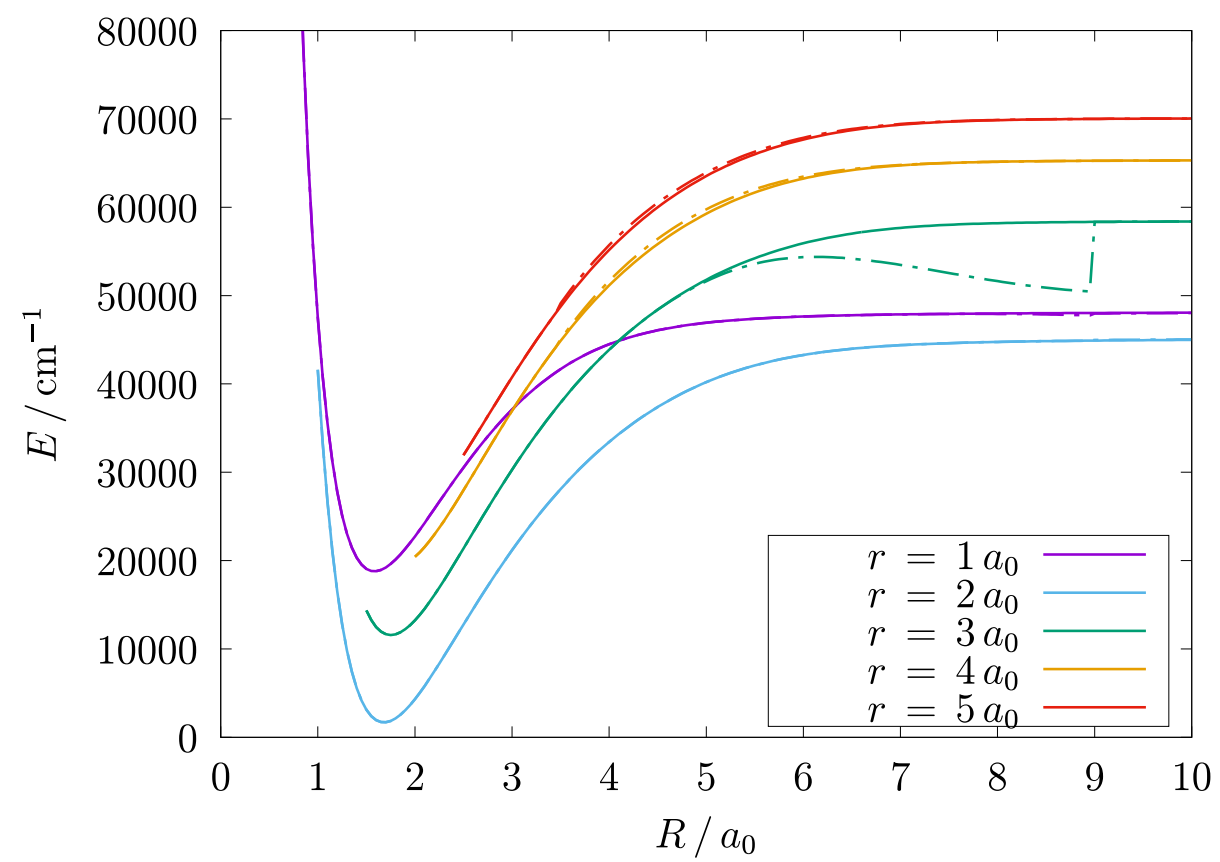

Figure 3. Comparison of some isosceles two-dimensional cuts of the new global ab initio BOPES75K for $\mathrm{H}_{3}^{+}$(solid lines) with the ones of the old GLH3P potential (dashed lines). Here the bond lengths of the $\mathrm{H}_{3}^{+}$molecule are $r_{1}=r, r_{2}=r_{3}=R$.

[32]. The vibrational ZPE for $\mathrm{H}_{3}^{+} E_{0}\left(\mathrm{H}_{3}^{+}\right)=4362.76 \mathrm{~cm}^{-1}$ was obtained from the large basis set calculation performed in Sec. 4 which is enough to converge the ZPE within $0.0001 \mathrm{~cm}^{-1}$. For comparison with experiment a further $64.21 \mathrm{~cm}^{-1}$ [33] must be added to the ZPE as the Pauli Principle means that the lowest allowed state of $\mathrm{H}_{3}^{+}$has is the $J=1, K=1$. The influence of disregarding non-BO effects is mainly conditioned by the adiabatic correction: the error from neglecting relativistic and QED effects is probably less than $1 \mathrm{~cm}^{-1}$ (as in calculations for the $\mathrm{H}_{2}$ molecule [34]), and the value of nonadiabatic correction is negligible at the dissociation threshold. Our results for the adiabatic correction at equilibrium give a value about $115.1 \mathrm{~cm}^{-1}$, whereas its value near dissociation was taken equal to be $114.5 \mathrm{~cm}^{-1}$ (see Sec. 2.2). Thus, our estimate of the uncertainty in our calculated dissociation energy is about $2 \mathrm{~cm}^{-1}$, and $D_{0}=35076 \pm 2 \mathrm{~cm}^{-1}=4.3489 \pm 0.0002 \mathrm{eV}$. This value is a little higher than the best previously-available theoretical result due to Lie \& Frye [35] of $4.337 \pm 0.002 \mathrm{eV}$. However, it remains lower than the best available experiment estimate of $D_{0}=4.381 \pm 0.021 \mathrm{eV}$ due to Cosby and Helm [36] . We believe our value is the best available estimate of the dissociation energy of $\mathrm{H}_{3}^{+}$. 

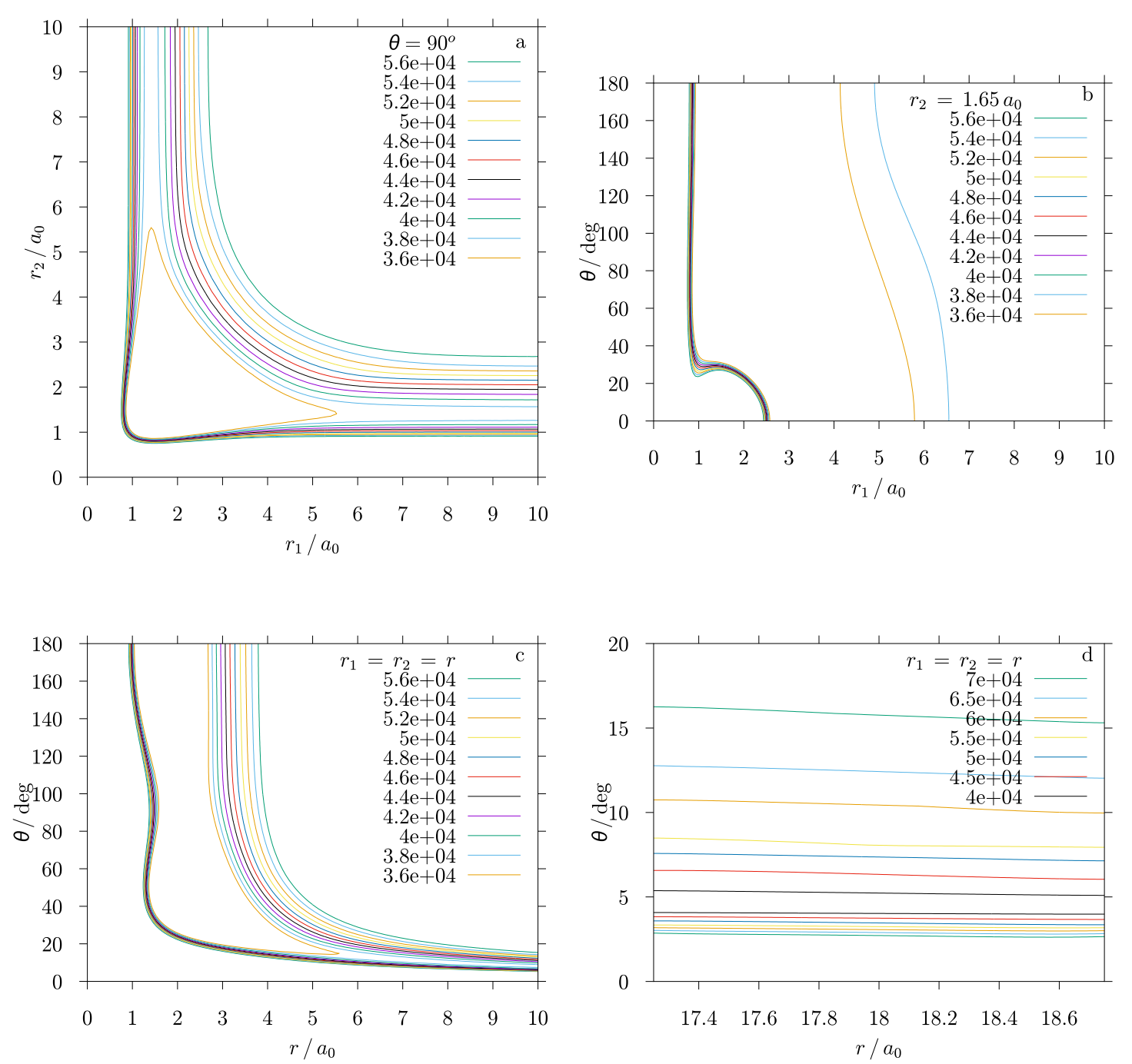

Figure 4. Contour plots of BOPES75K in the switching regions using $\mathrm{H}-\mathrm{H}$ bondlengths $\left(r_{1}, r_{2}\right)$ and the angle between them $(\theta)$ : (a) configurations with $\theta=90^{\circ}$ in the energy switching region from 36000 to $56000 \mathrm{~cm}^{-1}$; (b) configurations with $r_{2}=1.65 a_{0}$, in the same energy switching region; (c) isosceles configurations with $r_{1}=r_{2}=r$, in the same energy switching region; (d) isosceles configurations with $r_{1}=r_{2}=r$, for asymptotic switching region with $r$ varied from 17.25 to $18.75 a_{0}$. Energy values, which correspond to various isolines, are indicated on plots in $\mathrm{cm}^{-1}$.

\section{Nuclear motion calculations}

In order to test the new PES75K+ surface, calculations were performed using the DVR3D variational nuclear motion program suite [37]. The calculations were performed in Jacobi coordinates, and the discrete variable representation (DVR) grids were based on spherical oscillator functions for both the atom - diatom coordinate, $R$, and the diatomic coordinate, $r$, defined by the parameters $\alpha=0.0$ and $\omega_{e}=0.07$ atomic units [38]. A DVR in (associated) Legendre functions was used for the angular coordinate, $\theta$. The grids contained 60,58 , and 68 points for $R, r$ 
and $\theta$ coordinates, respectively. The final diagonalized matrices for the vibrational problem had a dimension of 20000 .

We compute vibrational energy levels, i.e. with total angular momentum $J=0$, using the new BOPES75K and the GLH3P PES for two cases: (i) for the surfaces in BO approximation using nuclear masses only - up to $60000 \mathrm{~cm}^{-1}$, and (ii) for these PESs augmented by adiabatic, relativistic and quantum electrodynamics correction surfaces, and allowing for non-adiabatic effects by using different effective vibrational and rotational masses as was suggested by Moss [39]. Calculations were performed up to the first dissociation limit of $\mathrm{H}_{3}^{+}$at about $37200 \mathrm{~cm}^{-1}$, and a bit above. This non-adiabatic model has been shown to give highly accurate predictions of $\mathrm{H}_{3}^{+}$spectra [40]. In the latter calculation set with BOPES75K the new global correction surfaces with exponential "tails" were used, and the vibrational mass was to $1.007517 \mathrm{Da}$ - a value intermediate between nuclear and atomic masses, which is the optimal one for the issue of the most accurate prediction of experimental vibrational band origins (see Table 6) and was obtained manually by trial-end-error method. For calculations with GLH3P adiabatic and relativistic correction surfaces[21], and a QED correction surface [31], which do not have global character, were used. In these calculations, the value of the vibrational mass derived by Moss for $\mathrm{H}_{2}^{+}$[39] and used previously for $\mathrm{H}_{3}^{+}$[21, 40] was also employed. For calculations with $J>0$ a rotational mass is also needed; this was always set to the proton (nuclear) mass, as before [21, 40].

The levels of $\mathrm{H}_{3}^{+}$included in the calculations of Table 4 consist of two distinct sets - bound and unbound levels. The bound levels have a clear physical meaning, the unbound ones - the levels above the dissociation, are artefacts of the chosen basis set. However, since the basis sets in both GLH3P and PES75K calculations are the same, the discrepancies between these artefact levels display the real difference between the resonances, which could be obtained using the same PESs and, for example, a complex absorbing potential (CAP) [41, 42].

A comparison between the vibrational energies obtained in the PES75K and GLH3P calculations is performed in Tables 4 and 5. One can see that there is a minor difference between the energies calculated on the basis of the two BO potentials up to the dissociation energy value, but significant differences (up to tens of $\mathrm{cm}^{-1}$ ) appear in energy range from about $40000 \mathrm{~cm}^{-1}$ and above. This is a direct consequence of fixing the unsmooth parts of the GLH3P PES.

Thus, Table 5 reflects only changes in the way non-BO effects are taken into 
account as it covers only the energy region up to $37000 \mathrm{~cm}^{-1}$. The difference between the approaches performed in the present work and previously in works by Pavanello et al. 21] and Lodi et al. [31] becomes significant for energies above $30000 \mathrm{~cm}^{-1}$, i.e. in the energy region where the old correction surfaces were not fitted accurately.

Table 4. Root mean square deviations ( $\mathrm{rms}$ ) and maximum absolute deviation ( $\mathrm{max}$ ) values in $\mathrm{cm}^{-1}$ of the vibrational (with $J=0$ ) Born-Oppenheimer energies obtained using the new global ab initio potential BOPES75K from the ones calculated with the GLH3P PES for different energy ranges. $N_{v}(E)$ gives the number of vibrational states given in each energy range.

\begin{tabular}{crrr}
\hline \hline Energy range $/ \mathrm{cm}^{-1}$ & $N_{v}(E)$ & $\mathrm{rms}$ & $\max$ \\
\hline $0-5000$ & 6 & 0.0000 & 0.0000 \\
$5000-10000$ & 21 & 0.0000 & 0.0001 \\
$10000-15000$ & 52 & 0.0001 & 0.0002 \\
$15000-20000$ & 110 & 0.0001 & 0.0007 \\
$20000-25000$ & 203 & 0.0004 & 0.0015 \\
$25000-30000$ & 331 & 0.0017 & 0.0067 \\
$30000-35000$ & 528 & 0.0070 & 0.0340 \\
$35000-40000$ & 776 & 0.0225 & 0.1924 \\
$40000-45000$ & 1043 & 0.2612 & 1.5575 \\
$45000-50000$ & 1310 & 1.8626 & 8.2676 \\
$50000-55000$ & 1583 & 3.8516 & 20.0573 \\
$55000-60000$ & 1832 & 4.4036 & 14.2542 \\
\hline \hline
\end{tabular}

Table 5. Root mean square deviations ( $\mathrm{rms}$ ) and maximum absolute deviation ( $\max$ ) values in $\mathrm{cm}^{-1} \mathrm{of} \mathrm{vibra-}^{-}$ tional (with $J=0$ ) energy levels obtained using the new global ab initio potential PES75K+ from the ones calculated with the GLH3P PES, for different energy ranges. Here adiabatic, relativistic, quantum electrodynamics and (partially) nonadiabatic effects were considered in following ways: for PES75K+ - as described above, for GLH3P PES - as in Refs. 21] (adiabatic, relativistic and nonadiabatic parts) and [31] (QED part). $N_{v}(E)$ gives the number of vibrational states given in each energy range.

\begin{tabular}{cccr}
\hline \hline Energy range $/ \mathrm{cm}^{-1}$ & $N_{v}(E)$ & $\mathrm{rms}$ & $\max$ \\
\hline $0-5000$ & 6 & 0.0770 & 0.0983 \\
$5000-10000$ & 21 & 0.1485 & 0.1885 \\
$10000-15000$ & 52 & 0.2059 & 0.2524 \\
$15000-20000$ & 110 & 0.2410 & 0.2844 \\
$20000-25000$ & 203 & 0.2175 & 0.3291 \\
$25000-30000$ & 331 & 0.1281 & 0.4733 \\
$30000-35000$ & 528 & 1.6869 & 7.0708 \\
$35000-38000$ & 432 & 3.5309 & 10.0955 \\
\hline \hline
\end{tabular}


Finally, in Table [6] a comparison of vibrational energy levels obtained using PES75K+ with adiabatic, relativistic, quantum electrodynamics, and (partially) nonadiabatic corrections with the available experimental data for states with $J=0$ is performed. The standard deviation obtained in this comparison is about $0.12 \mathrm{~cm}^{-1}$, which is almost twice smaller than the value $0.21 \mathrm{~cm}^{-1}$ obtained with vibrational masses used by Moss [39].

Table 6. Comparison of vibrational band origins (energy levels with $J=0$ ) obtained using the new global $a b$ initio potential PES75K+ with the available experimental data [43]. Adiabatic, relativistic, quantum electrodynamics and (partially) nonadiabatic effects were considered in this set of calculations. The calculated levels with symmetry $E^{\prime}$ were obtained as an arithmetic mean of (quasi-) degenerate even and odd pairs of DVR3D levels, which correspond to degenerate f-symmetry states. The experimental energies are supplemented by corresponding quantum numbers sets. Here $\left(\nu_{1}, \nu_{2}^{l_{2}}\right)$ are vibrational quantum numbers, $K$ is an absolute value of the projection of $J$ on the $\mathrm{C}_{3}$ axis and $G$ is an absolute value of quantum number $g=k-l_{2}$.

\begin{tabular}{lrrrrrrrr}
\hline \hline Symmetry & $E_{\text {exp }} / \mathrm{cm}^{-1}$ & PES75K+/cm & Obs. - calc. $/ \mathrm{cm}^{-1}$ & $K$ & $G$ & $\nu_{1}$ & $\nu_{2}$ & $l_{2}$ \\
\hline$E^{\prime}$ & 2521.411 & 2521.307 & 0.104 & 0 & 1 & 0 & 1 & 1 \\
$E^{\prime}$ & 4998.049 & 4997.912 & 0.137 & 0 & 2 & 0 & 2 & 2 \\
$E^{\prime}$ & 5554.060 & 5554.223 & -0.163 & 0 & 1 & 1 & 1 & 1 \\
$A_{2}^{\prime}$ & 7492.912 & 7492.807 & 0.106 & 0 & 3 & 0 & 3 & 3 \\
$E^{\prime}$ & 9113.080 & 9113.077 & 0.003 & 0 & 2 & 0 & 4 & 2 \\
$E^{\prime}$ & 10645.380 & 10645.338 & 0.042 & 0 & 2 & 2 & 2 & 2 \\
$E^{\prime}$ & 10862.910 & 10862.780 & 0.130 & 0 & 1 & 0 & 5 & 1 \\
$E^{\prime}$ & 11323.100 & 11323.145 & -0.045 & 0 & 1 & 3 & 1 & 1 \\
$E^{\prime}$ & 11658.400 & 11658.344 & 0.056 & 0 & 5 & 0 & 5 & 5 \\
$E^{\prime}$ & 12303.370 & 12303.376 & -0.006 & 0 & 1 & 2 & 3 & 1 \\
$E^{\prime}$ & 12477.380 & 12477.432 & -0.052 & 0 & 2 & 0 & 6 & 2 \\
$E^{\prime}$ & 13702.380 & 13702.676 & -0.296 & 0 & 1 & 0 & 7 & 1 \\
$E^{\prime}$ & 15122.810 & 15122.725 & 0.085 & 0 & 2 & 0 & 8 & 2 \\
\hline \hline
\end{tabular}

\section{Conclusions}

We present a modified global $\mathrm{H}_{3}^{+} \mathrm{PES}$ in both a simple $\mathrm{BO}$ form and as an augmented BO plus relativistic, QED and adiabatic corrections PES. The ab initio points used for this representation [24] are extremely accurate (accurate to an absolute energy of about $0.01 \mathrm{~cm}^{-1}$ ) and the only problem with the previous fit to these points was that analytical representation of some configurations with energy values above $37000 \mathrm{~cm}^{-1}$ was poor leading them to be excluded from the fit. The task of representing all the $a b$ initio points with the intrinsic accuracy of $a b$ initio 
calculations proved to be difficult, and the improved PESs presented here still do not recover the full accuracy of the underlying ab initio electronic structure points. Our new representation corrects the shortcomings of the previous incarnation [21] and displays the correct asymptotic behaviour.

Our new PES is suitable for a variety of calculations. Firstly, scattering calculations for collisions between protons and diatomic hydrogen molecules, particularly at ultra-low energies where such collisions are very sensitive to the details of the underlying PES. Secondly, the study of asymptotic vibrational states for the $\mathrm{H}_{3}^{+}$system [44]. Thirdly, for accurate calculation of $\mathrm{H}_{3}^{+}$resonance states, which should finally lead to the interpretation of the famous, indeed notorious, Carrington-Kennedy predissociation spectrum [45, 46].

\section{Acknowledgements}

This work was partially supported by the Russian Fund for Fundamental Studies as part of the research project \# 18-02-00705 and by the European Union's Horizon 2020 research and innovation programme under the Marie Sklodowska-Curie grant through grant number 701962. We thank Eryn Spinlove and Attila Csásár for comments on our potential energy surface.

\section{References}

[1] J. Tennyson, O.L. Polyansky, N.F. Zobov, A. Alijah and A.G. Császár, J. Phys. B: At. Mol. Opt. Phys. 50, 232001 (2017).

[2] T. Gonzalez-Lezana and P. Honvault, Intern. Rev. Phys. Chem. 33, 371 (2014).

[3] A. Carrington, J. Buttenshaw and R. Kennedy, Mol. Phys. 45, 753 (1982).

[4] A. Carrington and R.A. Kennedy, J. Chem. Phys. 81, 91 (1984).

[5] A. Carrington, J. Chem. Soc. Farad. T.2 82, 1089 (1986).

[6] A. Carrington and I.R. McNab, Acc. Chem. Res. 22, 218 (1989).

[7] A. Carrington, I.R. McNab and Y.D. West, J. Chem. Phys. 98, 1073 (1993).

[8] F. Kemp, C.E. Kirk and I.R. McNab, Phil. Trans. A 358 (1774), 2403 (2000).

[9] N.F. Zobov, O.L. Polyansky, C.R. Le Sueur and J. Tennyson, Chem. Phys. Lett. 260, 381 (1996).

[10] E. Carmona-Novillo, T. Gonzalez-Lezana, O. Roncero, P. Honvault, J.M. Launay, N. Bulut, F.J. Aoiz, L. Banares, A. Trottier and E. Wrede, J. Chem. Phys. 128, 014304 (2008).

[11] P. Honvault and Y. Scribano, J. Phys. Chem. A 117, 9778 (1997).

[12] M. Lara, P.G. Jambrina, F.J. Aoiz and J.M. Launay, J. Chem. Phys. 143, 204305 (2015).

[13] A. Ichihara and K. Yokoyama, J. Chem. Phys. 103, 2109 (1995). 
[14] R. Prosmiti, O.L. Polyansky and J. Tennyson, Chem. Phys. Lett. 273, 107 (1997).

[15] O.L. Polyansky, R. Prosmiti, W. Klopper and J. Tennyson, Mol. Phys. 98, 261 (2000).

[16] A. Aguado, O. Roncero, C. Tablero, C. Sanz and M. Paniagua, J. Chem. Phys. 112, 1240 (2000).

[17] L.P. Viegas, A. Alijah and A.J.C. Varandas, J. Chem. Phys. 126, 074309 (2007).

[18] L. Velilla, B. Lepetit, A. Aguado, J.A. Beswick and M. Paniagua, J. Chem. Phys. 129, 084307 (2008).

[19] R.A. Bachorz, W. Cencek, R. Jaquet and J. Komasa, J. Chem. Phys. 131, 024105 (2009).

[20] P. Barragan, R. Prosmiti, P. Villarreal and G. Delgado-Barrio, Int. J. Quant. Chem. 111, 368 (2011).

[21] M. Pavanello, L. Adamowicz, A. Alijah, N.F. Zobov, I.I. Mizus, O.L. Polyansky, J. Tennyson, T. Szidarovszky and A.G. Császár, J. Chem. Phys. 136, 184303 (2012).

[22] R. Jaquet and M.V. Khoma, J. Chem. Phys. 136, 154307 (2012).

[23] B. Mukherjee, S. Mukherjee and S. Adhikari, J. Phys. Conf. Ser. 759 (1), 012050 (2016).

[24] M. Pavanello, L. Adamowicz, A. Alijah, N.F. Zobov, I.I. Mizus, O.L. Polyansky, J. Tennyson, T. Szidarovszky, A.G. Császár, M. Berg, A. Petrignani and A. Wolf, Phys. Rev. Lett. 108, 023002 (2012).

[25] O.L. Polyansky, A. Alijah, N.F. Zobov, I.I. Mizus, R. Ovsyannikov, J. Tennyson, T. Szidarovszky and A.G. Császár, Phil. Trans. Royal Soc. London A 370, 5014 (2012).

[26] M. Pavanello and L. Adamowicz, J. Chem. Phys. 130, 034104 (2009).

[27] A.J.C. Varandas, J. Chem. Phys. 105, 3524 (1996).

[28] A.J.C. Varandas, J. Chem. Phys. 119, 2596 (2003).

[29] W.R. Gentry and C.F. Giese, Phys. Rev. A 11, 90 (1975).

[30] R.A. Bachorz, W. Cencek, R. Jaquet and J. Komasa, J. Chem. Phys. 131, 024105 (2009).

[31] L. Lodi, O.L. Polyansky, A.A. J. Tennyson and N.F. Zobov, Phys. Rev. A 89, 032505 (2014).

[32] K.K. Irikura, J. Phys. Chem. Ref. Data 36, 389 (2007).

[33] J. Ramanlal, O.L. Polyansky and J. Tennyson, Astron. Astrophys. 406, 383 (2003).

[34] M. Puchalski, J. Komasa, P. Czachorowski and K. Pachucki, Phys. Rev. Lett. 117, 263002 (2016).

[35] G.C. Lie and D. Frye, J. Chem. Phys. 96, 6784 (1992).

[36] P.C. Cosby and H. Helm, Chem. Phys. Lett. 152, 71 (1988).

[37] J. Tennyson, M.A. Kostin, P. Barletta, G.J. Harris, O.L. Polyansky, J. Ramanlal and N.F. Zobov, Comput. Phys. Commun. 163, 85 (2004).

[38] J. Tennyson and B.T. Sutcliffe, J. Mol. Spectrosc. 101, 71 (1983).

[39] R.E. Moss, Mol. Phys. 89, 195 (1996).

[40] O.L. Polyansky and J. Tennyson, J. Chem. Phys. 110, 5056 (1999).

[41] U.V. Riss and H.D. Meyer, J. Phys. B: At. Mol. Opt. Phys. 26, 4503 (1993).

[42] B.C. Silva, P. Barletta, J.J. Munro and J. Tennyson, J. Chem. Phys. 128, 244312 (2008).

[43] T. Furtenbacher, T. Szidarovszky, E. Mátyus, C. Fábri and A.G. Császár, J. Chem. Theory Comput. 9, 5471 (2013).

[44] J.J. Munro, J. Ramanlal and J. Tennyson, New J. Phys 7, 196 (2005). 
December 17, 2018

Molecular Physics final

Molecular Physics final
[45] A. Carrington, J. Buttenshaw and R.A. Kenedy, Mol. Phys. 45, 753 (1982).
[46] A. Carrington and R.A. Kennedy, J. Chem. Phys. 81, 91 (1984).

\begin{abstract}
[45] A. Carrington, J. Buttenshaw and R.A. Kenedy, Mol. Phys. 45, 753
[46] A. Carrington and R.A. Kennedy, J. Chem. Phys. 81, 91 (1984).

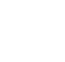

